\title{
PEDIATRAS E OS DISTÚRBIOS RESPIRATÓRIOS DO SONO NA CRIANÇA
}

\author{
*Aracy Pereira Silveira Balbani, Silke Anna Thereza Weber, Jair Cortez Montovani, Lídia Raquel de Carvalho \\ Trabalho realizado na Disciplina de Otorrinolaringologia e Cirurgia de Cabeça e Pescoço da Faculdade \\ de Medicina de Botucatu e Departamento de Bioestatística do Instituto de Biociências da \\ Universidade Estadual Paulista "Júlio de Mesquita Filho" (UNESP)
}

RESUMO - OBJETIVos. pesquisar as opiniões e condutas de pediatras frente aos distúrbios respiratórios do sono (DRS) em crianças.

Métodos. Foram selecionados aleatoriamente 516 pediatras do Estado de São Paulo. Foi enviado aos pediatras, por correio, um formulário de questões sobre: perfil profissional, seu conhecimento do DRS na criança, opinióes e condutas para diagnóstico e tratamento dessa doença.

Resultados. Retornaram preenchidos 112 questionários anônimos (21,7\%). 0 ensino de DRS na infância durante a graduação e a residência médica em Pediatria foi considerado insatisfatório, respectivamente, por $65,2 \%$ e $34,8 \%$ dos pediatras. Quarenta e nove pediatras $(43,8 \%)$ avaliaram seu conhecimento de DRS na criança como regular, $39(34,8 \%)$ como bom e 17 (I5,2\%) como insatisfatório. As questões de anamnese do sono consideradas mais importantes foram: respiração bucal, pausas respiratórias, número de horas de sono, sonolência diurna excessiva e chiado noturno. Os dados clínicos considerados mais importantes para a suspeita de síndrome da apnéia obstrutiva do sono (SAOS) foram: pausas respiratórias, hipertrofia da adenóide, respiração bucal, presença de anomalia craniofacial e ronco. As principais condutas citadas para diagnóstico de SAOS na criança foram: radiografia do cavum e avaliação com otorrinolaringologista $(25 \%)$ e oximetria de pulso noturna $(14,2 \%)$. Somente $I I, 6 \%$ dos pediatras indicaram a polissonografia de noite inteira e 4,5\%, a polissonografia breve diurna. As condutas consideradas mais eficazes para tratamento de DRS foram: cirurgias de adenoidectomia e adenotonsilectomia, orientação aos pais, perda de peso e higiene do sono.

Conclusöes. Há um descompasso entre as pesquisas sobre DRS na infância e sua abordagem na prática pediátrica.

Unitermos: Criança. Apnéia do sono tipo obstrutiva. Ronco. Educação médica.

\section{INTRODUÇÃo}

Crianças podem apresentar vários distúrbios respiratórios durante o sono, tais como 0 ronco primário, a apnéia e a hipopnéia!.

O ronco primário é o quadro clínico no qual há ruído respiratório, mas a arquitetura do sono, a ventilação alveolar e a saturação de oxigênio da hemoglobina mantêm-se normais. É freqüente na infância e afeta 7\% a 9\% das crianças de um a 10 anos!.

Apnéia central é a interrupção do comando do sistema nervoso central para a musculatura respiratória, cessando os movimentos tóraco-abdominais e o fluxo aéreo oronasal. Tem maior prevalência nos neonatos e prematuros. Apnéia obstrutiva é a interrupção do fluxo aéreo oronasal por colabamento das vias aéreas superiores (VAS), apesar do esforço da musculatura tóraco-abdominal. Nas crianças

\footnotetext{
*Correspondência:

Rua Capitão Lisboa, 715 - cj 33

CEP: 18270-070 - Tatuí - SP

Fax: (15) 3259-1152

E-mail: a_balbani@hotmail.com
}

normais é um evento raro durante o sono e não costuma durar mais do que 10 segundos. Apnéia mista envolve tanto a diminuição do comando respiratório central quanto a obstrução das VAS. Hipopnéia é a redução parcial do fluxo aéreo oronasal e também pode ser classificada como central, obstrutiva ou mista ${ }^{2,3}$.

Segundo a Classificação Internacional de Distúrbios do Sono, a síndrome da apnéia obstrutiva do sono (SAOS) é um "distúrbio intrínseco do sono caracterizado por episódios repetidos de obstrução das vias aéreas superiores associados à dessaturação da hemoglobina"4. Sua prevalência nas crianças varia de $0,7 \%$ a $3 \%$ nos diferentes estudos epidemiológicos ${ }^{4,5}$. O pico de incidência é observado nos pré-escolares, faixa etária na qual é mais comum a obstrução das vias aéreas superiores por hipertrofia das tonsilas palatinas ou da adenóide ${ }^{4,5}$.

Os estudos sobre distúrbios respiratórios do sono (DRS) na criança vêm se desenvolvendo muito nas últimas décadas. Atualmente sabe-se que a SAOS pode ter sérias conseqüências na infância: cor pulmonale, retardo do crescimento pôndero-estatural, alterações de comportamento, prejuízo do aprendizado, inteligência e outras funções cognitivas ${ }^{6}$. Contudo, parece haver um descompasso no diagnóstico e tratamento dessas afecções na prática clínica.

Owens $(2001)^{7}$ entrevistou 828 pediatras e médicos de família norte-americanos a respeito do DRS na infância. Cerca de 23\% não responderam corretamente a metade das questões de conhecimento do assunto. Eles não costumavam fazer anamnese sobre o sono em $42 \%$ dos pré-escolares, $52 \%$ dos escolares e $74 \%$ dos adolescentes durante as consultas de puericultura. Alguns motivos alegados foram: a) o médico deve aguardar que os pais relatem espontaneamente os problemas de sono na criança; b) perguntas sobre sono consomem o tempo da consulta que poderia ser dedicado a outros assuntos; c). a incidência de distúrbios do sono na criança é muito pequena e não justifica anamnese espećífica; d) o diagnóstico é irrelevante porque não há tratamentos eficazes para os distúrbios do sono na infância. Dentre esses médicos, 28,9\% nunca 
encaminharam uma criança para clínicas de sono; $53,2 \%$ raramente ou nunca pediam polissonografia aos pacientes e 63,6\% encaminhavam os casos suspeitos de SAOS diretamente para o otorrinolaringologista.

Chervin et al. $(2001)^{8}$ selecionaram 86 crianças com sintomas de distúrbios do sono (problemas respiratórios, movimentos periódicos das pernas, insônia ou sonolência diurna) atendidas em dois ambulatórios de Pediatria Geral nos Estados Unidos. Constataram que em menos de 10\% dos casos os médicos assistentes anotaram esses sintomas no prontuário da criança. Em apenas três casos foi descrita a realização de um tratamento específico do distúrbio de sono.

Considerando a importância do diagnóstico e do tratamento do DRS na criança, nosso objetivo foi pesquisar as opiniões e condutas de pediatras do Estado de São Paulo frente a essas afecções.

\section{Métodos}

Foram selecionados aleatoriamente no cadastro da Sociedade de Pediatria de São Paulo 516 pediatras do Estado. A esses especialistas foi enviado, nos meses de julho a setembro de 2003, por correio, um formulário padrão com questões sobre:

a) perfil do profissional: sexo, graduação em faculdade pública ou privada, tempo decorrido da formatura, titulação acadêmica, local de trabalho (município e instituição) e atividade exercida (Pediatria geral ou subespecialidade);

b) sua opinião a respeito do ensino de distúrbios respiratórios do sono (DRS) na criança durante o curso de graduação e a residência médica em Pediatria (excelente, bom, regular ou insatisfatório);

c) auto-avaliação do conhecimento atual do profissional sobre DRS na criança (excelente, bom, regular ou insatisfatório);

d) freqüência das queixas dos pais e da realização de anamnese do sono nas consultas de puericultura (sempre, freqüentemente, raramente ou nunca). Esses itens foram subdivididos nas diferentes faixas etárias: lactentes ( 0 a 2 anos de idade), pré-escolares (3 a 6 anos), escolares (7 a 14 anos) e adolescentes ( 15 a 20 anos);

e) relevância (muito importante, pouco importante ou sem importância) das seguintes perguntas para anamnese do sono na criança: hora em que a criança vai dormir; número de horas de sono; cochilos diurnos; sonolência diurna excessiva; sonilóquio; sonambulismo; insônia; pesadelos/terror noturno; criança dorme no quarto/cama dos pais; ronco; pausas respiratórias; respiração bucal; chiado noturno; tosse noturna; sono agitado; sudorese noturna; enurese noturna; encoprese noturna; ranger de dentes (bruxismo) e iluminação do quarto de dormir;

f) relevância (muito importante, pouco importante ou sem importância) dos seguintes dados clínicos para levantar a suspeita de DRS na criança: ronco; pausas respiratórias; sonolência diurna excessiva; obstrução nasal; respiração bucal; distúrbio de comportamento (hiperatividade); mau desempenho escolar; hipertrofia das amígdalas (tonsilas); hipertrofia da adenóide; rinite alérgica; obesidade; síndromes genéticas; anomalia craniofacial; cor pulmonale; retardo de crescimento e antecedente familiar de apnéia;

g) exames ou avaliações que o profissional solicita para investigar e confirmar o diagnóstico de síndrome da apnéia obstrutiva do sono (SAOS) na criança: avaliação com neuropediatra; avaliação com otorrinolaringologista; hemograma; nasofibroscopia; eletrencefalograma; radiografia do cavum; tele-radiografia lateral; eletrocardiograma; ecocardiograma; oximetria de pulso durante o sono; polissonografia de noite inteira; polissonografia breve diurna (triagem), outro exame (devendo especificálo) ou a alternativa "não solicita exames, pois o diagnóstico é clínico". Foi aceita mais de uma resposta a essa questão.

h) eficácia das seguintes opções de tratamento da SAOS na criança: orientação aos pais; higiene do sono; adenoamigdalectomia (adenotonsilectomia); adenoidectomia; amigdalectomia (tonsilectomia); perda de peso; descongestionantes nasais; corticosteróides nasais; corticosteróides sistêmicos; estimulantes (cafeína, doxapram, etc.); CPAP nasal (Continuous Positive Airway Pressure) / BiPAP (Bilevel Positive Airway Pressure); traqueostomia e observação apenas. Cada opção foi classificada como muito eficaz, pouco eficaz ou ineficaz. i) opinião sobre a adenoidectomia, tonsilectomia e adenotonsilectomia para tratamento do ronco e da apnéia obstrutiva do sono nas crianças (questão dissertativa).

Os questionários preservaram o anonimato dos participantes. Os dados foram submetidos à análise estatística pelo teste do Qui-quadrado através do programa de computador SAS versão 6.12, adotando-se 0 nível de significância de 5\% $(p<0,05)$.

\section{Resultados}

Treze questionários remetidos (2,5\%) retornaram porque os correios não localizaram os destinatários. Foram devolvidos preenchidos I I 2 dos 503 questionários entregues (22,2\%).

\section{a) Perfil dos participantes do estudo}

Dos 1 I 2 participantes, 67 (59,8\%) eram do sexo feminino e $45(40,2 \%)$ do masculino. Sessenta e nove $(61,6 \%)$ cursaram medicina em instituição pública de ensino superior; 41 (36,6\%) freqüentaram instituição privada e dois $(1,8 \%)$ não informaram esse dado. Cinqüenta e oito pediatras $(51,8 \%)$ haviam se formado há mais de 15 anos; 18 (16\%) há I II5 anos; 13 (11,6\%) há 5-10 anos e seis $(5,5 \%)$ há menos de cinco anos. Dezessete $(15,1 \%)$ não informaram o ano de formatura.

A distribuiçãa segundo a titulação acadêmica foi: 89 médicos $(79,5 \%)$ possuíam residência em Pediatria $(p<0,00 \mathrm{l})$; nove $(8 \%)$, mestrado; oito (7,1\%), doutorado; um $(0,9 \%)$, pós-doutorado e dois $(1,8 \%)$, livredocência. Três pediatras (2,7\%) não informaram a titulação acadêmica.

Dentre os entrevistados, 20 (18\%) trabaIhavam na capital do Estado; 42 (37,8\%) em município com mais de 500.000 habitantes; 14 (I2,6\%) em município com 100.000-500.000 habitantes; 10 (9\%) em município com 50.000-99.999 habitantes e $12(10,8 \%)$ em município com menos de 50.000 habitantes. Treze pediatras ( I I,7\%) trabalhavam em dois ou mais municípios e um $(0,9 \%)$ não respondeu à questão.

Dezesseis pediatras (14,4\%) trabalhavam em um único local - sete em instituição universitária (6,3\%), cinco em consultório particular (4,5\%), três em posto/centro de saúde (2,7\%) e um em entidade assistencial (0,9\%). Cinqüenta e três (47,7\%) trabalhavam em dois locais; 31 (28\%) em três locais e II (9,9\%) em quatro locais. Um pediatra não informou esse dado. 


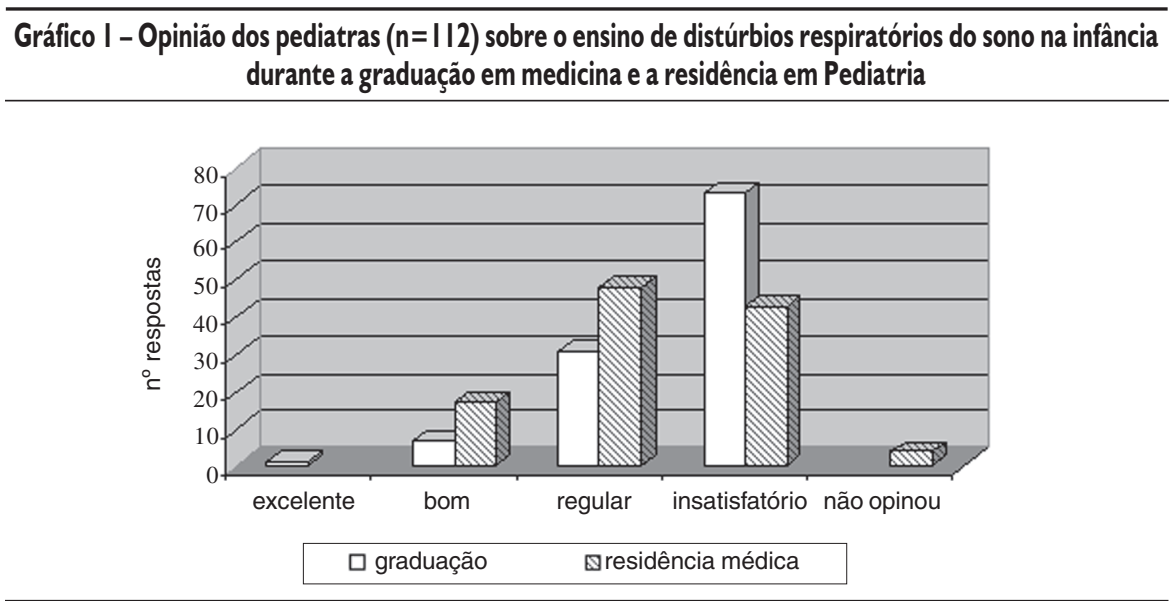

Gráfico 2 - Freqüência dos sintomas respiratórios noturnos durante as consultas de puericultura nas diferentes faixas etárias

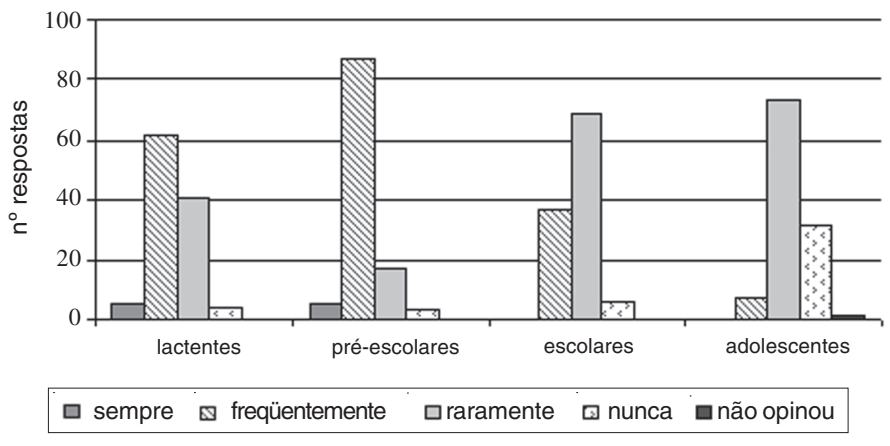

Gráfico 3-Freqüência da anamnese do sono durante as consultas de puericultura nas diferentes faixas etárias

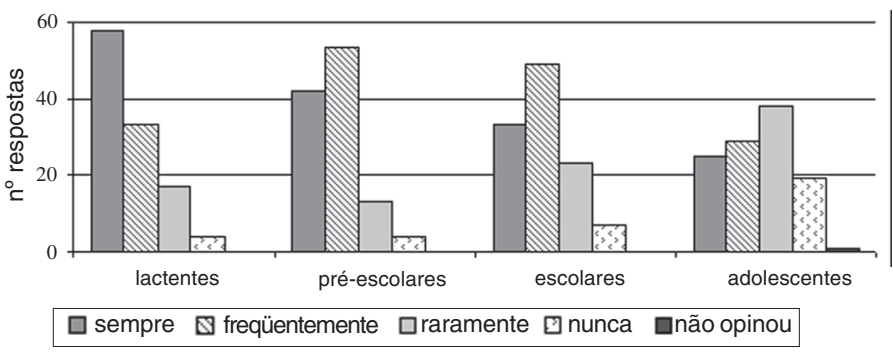

Foram mencionadas 18 áreas de atividade, sendo as mais freqüentes: Pediatria Geral (65 profissionais, 58\%), Neonatologia ( 3 profissionais, I 1,6\%), Terapia Intensiva (sete profissionais, $6,3 \%$ ) e Pneumologia (cinco profissionais, 4,5\%). no de DRS na infância durante a graduação, e 3 I $(27,7 \%)$ consideraram-no regular. Sete avaliaram o ensino de DRS como bom, dos quais três cursaram faculdade particular e quatro, faculdade pública. $O$ ensino de DRS na residência médica em Pediatria foi apontado como regular por 48 profissionais (42,9\%) e insatisfatório por 43 (38,4\%).

\section{c) Auto-avaliação do conhecimento atu-} al do profissional sobre DRS na criança

Quarenta e nove pediatras (43,8\%) avaliaram seu conhecimento atual de DRS na criança como regular, 39 (34,8\%) como bom, 17 (15,2\%) como insatisfatório e dois (।,8\%) como excelente - ambos possuíam residência médica, sendo um pediatra geral e um homeopata. Cinco pediatras (4,5\%) não responderam à questão.

\section{d) Freqüência das queixas dos pais e da realização de anamnese do sono nas consultas de puericultura}

A freqüência das queixas de sintomas respiratórios noturnos nas diferentes faixas etárias é mostrada no Gráfico 2. Segundo 87 pediatras $(77,7 \%)$, as queixas dos pais ocorrem freqüentemente nas consultas realizadas nos pré-escolares. Já nas consultas dos adolescentes, tais queixas ocorrem raramente, de acordo 73 pediatras $(65,2 \%)$, ou nunca, segundo 3 I profissionais $(27,2 \%)$.

A freqüência de realização de anamnese do sono é vista no Gráfico 3. Quatro pediatras (3,6\%) nunca fazem essa anamnese nas consultas de puericultura dos lactentes e pré-escolares; sete $(6,3 \%)$ nunca a fazem ao atender escolares e 19 (17\%) nunca a fazem ao atender adolescentes. Três pediatras informaram que não atendem adolescentes no seu dia-a-dia.

\section{e) Relevância das questões para anamnese do sono na criança}

As questões consideradas muito importantes pelo maior número de profissionais $(p<0,001)$ foram: respiração bucal (103 pediatras, 92\%), pausas respiratórias ( 100 pediatras, 89,3\%), número de horas de sono (96 pediatras, $85,7 \%)$, sonolência diurna excessiva e chiado noturno (95 pediatras, 84,8\%).

As questões tidas como sem importância pelo maior número de profissionais foram: encoprese noturna (14 pediatras, $12,5 \%$ ), iluminação do quarto de dormir 


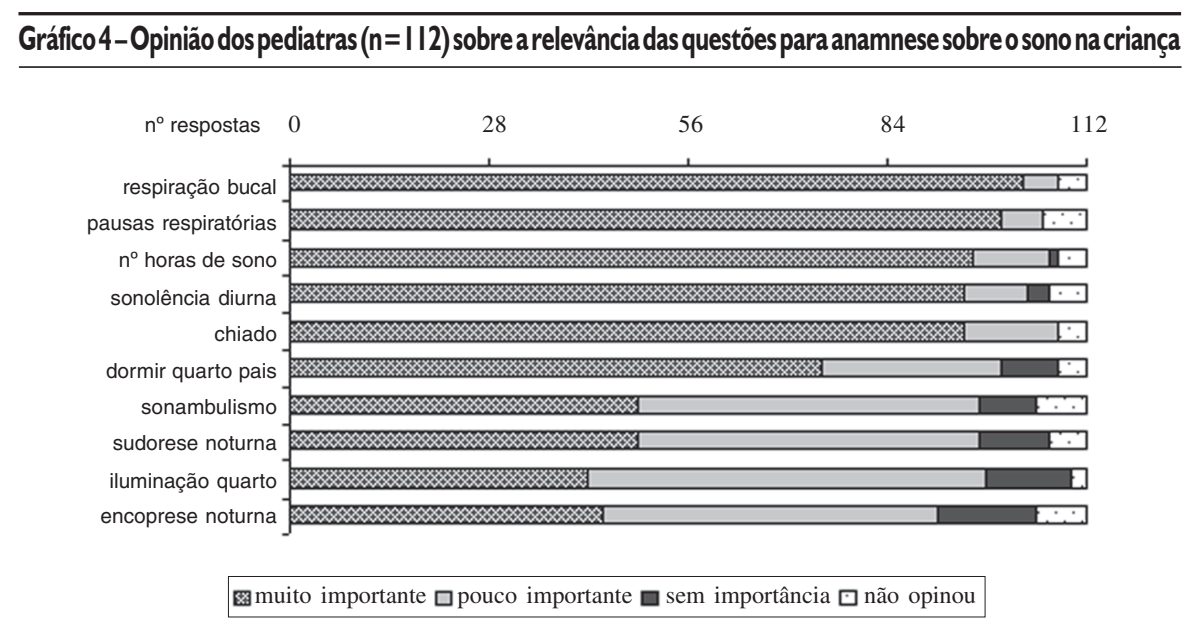

Gráfico 5 - Opinião dos pediatras ( $n=$ I I2) sobre a relevância dos dados clínicos para suspeita de apnéia obstrutiva do sono na criança

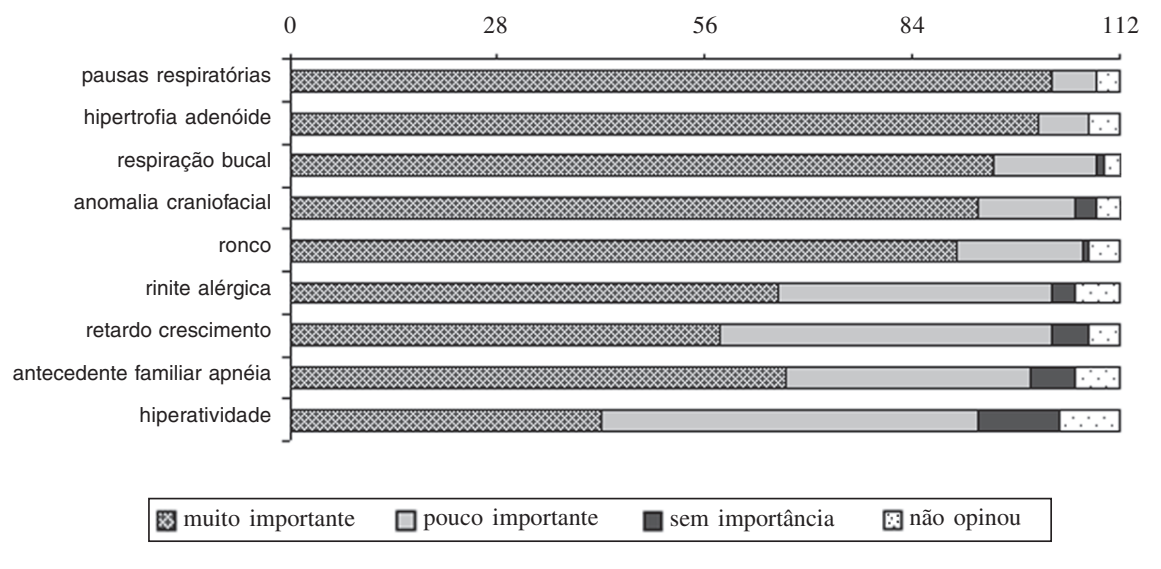

\begin{tabular}{|c|c|c|c|c|}
\hline \multicolumn{5}{|c|}{$\begin{array}{l}\text { Tabela I - Opinião dos pediatras }(n=\mid I 2) \text { sobre a eficácia das opções de tratamento } \\
\text { da síndrome da apnéia obstrutiva do sono na criança }\end{array}$} \\
\hline Tratamento & Muito eficaz & Pouco eficaz & Ineficaz & Não opinou \\
\hline$\overline{\text { Adenoidectomia }}$ & $75(67,0 \%)$ & $\overline{17(15,2 \%)}$ & $\overline{-}$ & $20(17,8 \%)$ \\
\hline Adenotonsilectomia & $73(65,2 \%)$ & $20(17,8 \%)$ & I $(0,9 \%)$ & $18(16,1 \%)$ \\
\hline Orientação aos pais & $63(56,3 \%)$ & $35(31,2 \%)$ & $3(2,7 \%)$ & || (9,8\%) \\
\hline Perda de peso & $63(56,3 \%)$ & $31(27,7 \%)$ & $5(4,4 \%)$ & $13(1 \mid, 6 \%)$ \\
\hline Higiene do sono & $59(52,7 \%)$ & $38(33,9 \%)$ & $2(1,8 \%)$ & $13(11,6 \%)$ \\
\hline Tonsilectomia & $44(39,2 \%)$ & $33(29,5 \%)$ & $5(4,5 \%)$ & $30(26,8 \%)$ \\
\hline CPAPnasal/BiPAP & $37(33,0 \%)$ & $15(13,4 \%)$ & $15(13,4 \%)$ & $45(40,2 \%)$ \\
\hline Corticóides nasais & $29(25,9 \%)$ & $56(50,0 \%)$ & $12(10,7 \%)$ & $15(13,4 \%)$ \\
\hline Traqueostomia & $19(17,0 \%)$ & || $(9,8 \%)$ & $37(33,0 \%)$ & $45(40,2 \%)$ \\
\hline Corticóides sistêmicos & $15(13,4 \%)$ & $53(47,3 \%)$ & $26(23,2 \%)$ & $18(16,1 \%)$ \\
\hline Descongestionantes nasais & $8(7,1 \%)$ & $50(44,5 \%)$ & $40(35,7 \%)$ & $14(12,5 \%)$ \\
\hline Estimulantes (cafeína, etc.) & $6(5,4 \%)$ & $36(32,1 \%)$ & $36(32,1 \%)$ & $34(30,4 \%)$ \\
\hline Observação apenas & $2(1,8 \%)$ & $16(\mid 4,2 \%)$ & $62(55,4 \%)$ & $32(28,6 \%)$ \\
\hline
\end{tabular}

( 12 pediatras, 10,7\%), sudorese noturna (10 pediatras, 8,9\%), sonambulismo e se a criança dorme no quarto/cama dos pais (8 pediatras, 7, 1\%). O Gráfico 4 representa a relevância dessas questões segundo as opiniões dos profissionais.

\section{f) Relevância de dados clínicos para levantar a suspeita de SAOS na criança}

Os dados clínicos considerados muito importantes pela maioria dos pediatras $(p<0,00$ I) foram: ocorrência de pausas respiratórias (103 profissionais, 92\%), hipertrofia da adenóide ( 101 profissionais, 90,2\%), respiração bucal (95 profissionais, 84,8\%), presença de anomalia craniofacial (93 profissionais, $83 \%$ ) e ronco (90 profissionais, 90,4\%).

Os dados clínicos considerados sem importância pelo maior número de profissionais $(p<0,00$ I $)$ foram: distúrbio de comportamento (I I pediatras, $9,8 \%)$, antecedente familiar de apnéia (6 pediatras, 5,4\%), retardo de crescimento ( 5 pediatras, 4,5\%), mau desempenho escolar, rinite alérgica e síndromes genéticas (3 pediatras, 2,7\%). O Gráfico 5 representa a relevância desses dados clínicos segundo as opiniões dos profissionais.

\section{g) Exames/avaliações solicitados pelo pediatra para investigação e confirmação diagnóstica de SAOS na criança}

Foram relatadas 45 condutas diferentes para diagnóstico da SAOS na criança. Vinte e oito pediatras (25\%) solicitam radiografia do cavum e avaliação com otorrinolaringologista; 13 (1 1,6\%) solicitam, além dos exames anteriores, também a nasofibroscopia para avaliação das vias aéreas superiores. Seis $(5,4 \%)$ encaminham a criança diretamente para o otorrinolaringologista.

Dezesseis profissionais (14,2\%) solicitam que a criança seja submetida à oximetria de pulso noturna. Treze (1।,6\%) pedem polissonografia de noite inteira e cinco (4,5\%), polissonografia breve diurna.

Quanto à familiaridade com os critérios polissonográficos para diagnóstico de apnéia, hipopnéia e ronco primário na criança, um pediatra $(0,9 \%)$ considerou-a excelente, dois $(1,8 \%)$ consideraram-na boa, 21 (18,8\%), regular e 83 (74, I\%), insatisfatória $(p<0,001)$. Cinco pediatras não responderam.

\section{h) Opinião sobre a eficácia das opções de tratamento da SAOS na criança}

As opiniões são mostradas na Tabela I. 


\section{i) Opinião sobre a adenoidectomia, tonsilectomia e adenotonsilectomia para tratamento de ronco e apnéia obstrutiva do sono em crianças}

Cinqüenta e quatro pediatras (48,2\%) consideram as cirurgias de adenoidectomia, tonsilectomia ou adenotonsilectomia o tratamento de eleição para os DRS causados por hipertrofia do tecido linfático faríngeo. Dezenove (17\%) acham que as cirurgias devem ser indicadas somente se há falha do tratamento clínico da rinite e obesidade. Seis (5,4\%) são contrários a esses tratamentos cirúrgicos (três acreditam que há recidiva da hipertrofia do tecido linfático, e um homeopata diz que as cirurgias são "mutilantes" e "pioram a alergia respiratória"). Cinco (4,5\%) vêem as cirurgias com restrições, por acreditarem que a melhora dos sintomas da criança é apenas temporária. Dezoito profissionais $(16,1 \%)$ não opinaram, dos quais dois alegaram "falta de conhecimento".

Destacamos alguns comentários dos pediatras: "Pacientes não obesos respondem bem à adenotonsilectomia". "Só indico cirurgia nas crianças maiores de três anos". "Se há apnéia considero a cirurgia mesmo se a criança for menor de três anos de idade". "Não indico cirurgia aos casos de ronco, somente aos de apnéia comprovada". "Deixo a critério do otorrinolaringologista a indicação da cirurgia". "Se há causas multifatoriais - alergia, obesidade - a cirurgia não deve ser o único tratamento". "A única experiência que tive foi o relato de uma mãe, que não sentiu melhora no quadro da filha após adenoamigdalectomia". "Acredito apenas na adenoamigdalectomia".

Uma pediatra acredita que os otorrinolaringologistas "postergam demais" o tratamento cirúrgico das crianças com DRS, enquanto outro alerta para a importância do "tratamento fonoaudiológico e ortodôntico" nas crianças respiradoras bucais.

\section{Discussão}

Constatamos neste trabalho que o ensino dos distúrbios respiratórios do sono (DRS) na infância durante a graduação em medicina e a residência em Pediatria foi insatisfatório, respectivamente, para 65,5\% e $38,4 \%$ de uma amostra de pediatras do
Estado de São Paulo. Essa deficiência do ensino médico refletiu-se na auto-avaliação dos profissionais entrevistados: $43,8 \%$ julgam regular seu conhecimento do assunto e 15,2\%, insatisfatório. Um participante do estudo comentou que teve as primeiras informações sobre DRS em um curso de Medicina do Tráfego, no qual havia "um protocolo para investigação clínica dos DRS em adultos", o que lhe "chamou a atenção" para avaliar essas afecções nas crianças, "após 20 anos de profissão".

Para $77,7 \%$ dos pediatras, os pais queixam-se freqüentemente de DRS nos préescolares durante as consultas de puericultura. Esse resultado é compatível com os dados de literatura sobre a maior prevalência de ronco primário e síndrome da apnéia obstrutiva do sono (SAOS) nessa faixa etária ${ }^{4,5}$.

Comparando nossos resultados com os de Owens $(200 \text { I) })^{7}$ e Chervin et al. $(200 \text { I) })^{8}$, observamos uma freqüência muito menor de pediatras que não fazem anamnese do sono na criança durante a puericultura: apenas 3,6\% nas consultas de pré-escolares, $6,3 \%$ nas consultas de escolares e $17 \%$ nas consultas de adolescentes. Ainda assim, enfatizamos que a anamnese é um passo fundamental para o diagnóstico dos distúrbios do sono na criança e no adolescente.

Algumas das questões de anamnese do sono mais importantes para os pediatras são justamente sobre o padrão respiratório da criança: respiração bucal, pausas respiratórias e chiado noturno. A sonolência diurna excessiva também foi considerada um dado muito importante por $84,8 \%$ dos médicos, mas lembramos que, ao contrário dos adultos, menos de $20 \%$ das crianças com apnéia obstrutiva apresentam despertares corticais e fragmentação do sono. Assim, a sonolência diurna não é um dos principais sintomas da doençç ${ }^{3,9,10}$. Por outro lado, as pesquisas mostram que sono agitado, sudorese e enurese noturnas podem ser manifestações da SAOS na criança4.

Distúrbios de comportamento, retardo de crescimento e mau desempenho escolar foram considerados pela maioria dos pediatras como dados clínicos sem importância para levantar a suspeita de SAOS. Porém, vários trabalhos comprovam o impacto negativo dos DRS na cognição, aprendizado e comportamento da criança ${ }^{1 /-13}$. Há evidência de que os DRS acometem I/5 a I/3 das crianças com déficit de atenção e distúrbio de hiperatividade". Além disso, pode ocorrer retardo de crescimento nas crianças com SAOS em razão da menor secreção de hormônio de crescimento $(\mathrm{GH})$ durante os estágios profundos do sono de ondas lentas ${ }^{14}$. Portanto, é preciso que pediatras, neuropediatras e outros especialistas cogitem a hipótese de DRS nas crianças com déficit de crescimento ou alterações do comportamento e aprendizagem.

A polissonografia em laboratório de sono durante uma noite inteira é o padrãoouro para diagnóstico da SAOS ${ }^{15}$, embora ainda não estejam normatizados os critérios de interpretação do exame em crianças e adolescentes. Seus principais parâmetros são: o índice de apnéia $(I A$, número de apnéias obstrutivas e mistas por hora de sono) e o índice de apnéia e hipopnéia (IAH, número de apnéias e hipopnéias obstrutivas e mistas por hora de sono $)^{2,9,16}$. A recomendação atual da "American Thoracic Society" é de que $|A H<|$ é diagnóstico de ronco primário, enquanto $\mathrm{IA}$ ou $\mathrm{IAH}=$ I são diagnósticos de SAOS na criançal' ${ }^{16}$.

Verificamos que somente 11,6\% dos pediatras solicitam a polissonografia de noite inteira, e 4,5\% pedem a polissonografia breve diurna para confirmar o diagnóstico de SAOS. Vimos também que 18,8\% consideram regular sua familiaridade com os critérios diagnósticos do exame, e 74, I \% não se consideram familiarizados. Acreditamos que isso decorra das restrições para realizar a polissonografia na prática diária, pois o exame está disponível em poucos locais geralmente grandes cidades ou centros de pesquisa em medicina do sono - e seu custo é alto. Essa dificuldade leva os pediatras a diagnosticarem SAOS com base na sua experiência clínica e em outros exames mais acessíveis.

Vinte e cinco por cento dos pediatras pedem a radiografia do cavum e a avaliação do otorrinolaringologista e $14,2 \%$, a oximetria de pulso noturna. Estudos científicos apontam que a oximetria de pulso noturna é um bom meio de triagem dos casos suspeitos de SAOS, mas não substitui 
a polissonografia para diagnóstico, pois algumas crianças com a doença podem ter oximetria normal ${ }^{1,4,15}$.

A nasofibroscopia, mencionada por I I,6\% dos pediatras, é muito útil no exame das cavidades nasais e da rinofaringe pelo otorrinolaringologista. Souza et al. (2000) $)^{17}$ encontraram hipertrofia da adenóide através da nasofibroscopia em 4I crianças cuja radiografia do cavum teve resultado normal.

Destacamos que 63,6\% dos médicos participantes do estudo de Owens encaminham a criança com DRS diretamente para o otorrinolaringologista, enquanto apenas 5,4\% dos pediatras entrevistados por nós adotam essa conduta.

As medidas consideradas mais eficazes para tratamento dos DRS foram: as cirurgias de adenoidectomia e adenotonsilectomia, a orientação aos pais, a perda de peso e a higiene do sono da criança. Dentre os participantes do estudo, 48,2\% acreditam que essas cirurgias são o tratamento de eleição para a SAOS provocada por hipertrofia adenotonsilar. Segundo a literatura, a adenotonsilectomia permite curar a SAOS em mais de $75 \%$ das crianças com hipertrofia adenotonsilar significativa, mesmo que sejam obesas' ${ }^{15}$. Sabe-se que há maior risco de complicações respiratórias no pós-operatório de adenotonsilectomia em algumas crianças com SAOS (lactentes, portadoras de anomalias craniofaciais, síndromes genéticas, doenças neuromusculares, doença pulmonar obstrutiva crônica, cor pulmonale ou anemia falciforme e naquelas com $\mid \mathrm{AH}>10 /$ hora) ${ }^{15,16}$. Assim, o otorrinolaringologista deve estar adequadamente preparado para auxiliar no diagnóstico e tratamento dos casos de DRS na criança, sempre em sintonia com o pediatra.

A pressão positiva contínua das vias aéreas (CPAP ou BiPAP nasal) foi avaliada como muito eficaz por $33 \%$ e ineficaz por 13,4\% dos pediatras. Seu uso é recomendado nos casos de SAOS em que: I. não há hipertrofia adenotonsilar (doenças neurológicas e musculares, p. ex.); 2. a criança tem contra-indicações para a cirurgia de adenotonsilectomia, 3. a apnéia obstrutiva persiste no pós-operatório ou 4. a SAOS é severa e requer tratamento paliativo até que a criança seja operada ${ }^{15}$. De acordo com os trabalhos consultados, O CPAP beneficia muito essas crianças ${ }^{3,15}$.

Com base nesse panorama, sugerimos maior ênfase no ensino dos distúrbios respiratórios do sono na infância durante a formação do médico generalista e do pediatra. Também são desejáveis cursos de atualização em programas de educação continuada para os profissionais que já concluíram a residência médica em Pediatria, colocando-os a par dos avanços científicos na área. A capacitação do pediatra e demais especialistas envolvidos no diagnóstico e tratamento dos DRS permitirá oferecer a essas crianças um atendimento cada vez melhor.

\section{Conclusóes}

A entrevista com I I 2 pediatras do Estado de São Paulo a respeito dos distúrbios respiratórios do sono (DRS) na criança revelou que:

a) o ensino de DRS na infância foi considerado insatisfatório na graduação e na residência em Pediatria, respectivamente, por $65,2 \%$ e $38,4 \%$ dos participantes

b) 49 profissionais $(43,8 \%)$ avaliaram seu conhecimento dos DRS na criança como regular, 39 (34,8\%) como bom, 17 ( I 5,2\%) como insatisfatório;

c) as questões de anamnese do sono na criança consideradas mais importantes foram: respiração bucal, pausas respiratórias, número de horas de sono, sonolência diurna excessiva e chiado noturno;

d) os dados clínicos considerados mais importantes para a suspeita de síndrome da apnéia obstrutiva do sono (SAOS) foram: pausas respiratórias, hipertrofia da adenóide, respiração bucal, presença de anomalia craniofacial e ronco;

e) as principais condutas citadas para diagnóstico de SAOS na criança foram: radiografia do cavum e avaliação com otorrinolaringologista (25\%) e oximetria de pulso noturna ( $14,2 \%)$;

f) as medidas consideradas mais eficazes para tratamento dos DRS foram: as cirurgias de adenoidectomia e adenotonsilectomia, a orientação aos pais, a perda de peso e a higiene do sono da criança.

\section{Agradecimentos}

Os autores agradecemà Sociedade de Pediatria de São Paulo e aos médicos pediatras que gentilmente participaram do estudo.

\section{Conflito de interesse: não há.}

\section{SUMMARY}

Pediatricians and sleep-disordered BREATHING IN THE CHILD

OBJECTIVES. assessment of opinions and practices of pediatricians concerning sleepdisordered breathing (SDB) in children.

Methods. randomly 516 pediatricians were selected in the state of São Paulo, Brazil. A survey mailed to them included questions regarding: their professional profile, knowledge about SDB in childhood, opinions and practices for diagnosis and treatment of these diseases.

RESULTS. I/2 anonymous completed surveys were returned (21.7\%). The teaching of SDB during medical school and pediatric residency training was considered unsatisfactory respectively by $65.2 \%$ and $34.8 \%$ of the pediatricians. Forty-nine respondents $(43.8 \%)$ rated their knowledge about SDB in children as regular, 39 (34.8\%) as good and 17 (15.2\%) as unsatisfactory. The most important sleep-related questions were: mouth breathing, breathing pauses, sleep amount, excessive daytime sleepiness and nocturnal wheezing. Clinical aspects regarded as the most significant for suspecting obstructive sleep apnea syndrome (OSAS) were: breathing pauses, adenoid hypertrophy, mouth breathing, craniofacial anomaly and snoring. The most frequent practices for evaluation of OSAS in children were: cavum radiography with referral to an otorhinolarnygologist (25\%) and nocturnal pulse oximetry (14.2\%). Only 11.6\% of pediatricians recommended overnight polysomnography and $4.5 \%$, nap polysomnography. The most effective practices for SDB were considered to be: adenoidectomy and adenotonsillectomy, parents counseling, weight loss and sleep hygiene.

ConClusions. there is a gap between research on SDB in childhood and pediatric practice. [Rev Assoc Med Bras 2005; 5 I (2): 80-6]

KEY wORDS: Child. Obstructive sleep apnea. Snoring. Medical education. 


\section{RefERÊNCIAS}

I. Anstead M. Pediatric sleep disorders: new developments and evolving understanding. Curr Opin Pulm Med 2000;6:50 I -6.

2. Marcus CL, Omlin KJ, Basinki DJ, Bailey SL, Rachal AB, Von Pechmann WS, et al. Normal polysomnographic values for children and adolescents. Am Rev Respir Dis 1992;146: 1235-9.

3. Marcus CL. Sleep-disordered breathing in children. Am J Respir Crit Care Med 200I; 164:16-30.

4. Schechter MS. Technical report: diagnosis and management of childhood obstructive sleep apnea. Pediatrics 2002; 109:e69.

5. Brunetti L, Rana S, Lospalluti ML, Pietrafesa A, Francavilla R, Fanelli $M$, et al. Prevalence of obstructive sleep apnea in a cohort of 1207 children of Southern Italy. Chest 200 I; I 20: 1930-5.

6. Lipton AJ, Gozal D. Treatment of obstructive sleep apnea in children: do we really know how? Sleep Med Rev 2003;7:61-80.

7. Owens JA. The practice of pediatric sleep medicine: results of a community survey. Pediatrics 200 I; I 08:e5 I.

8. Chervin RD, Archbold KH, Panahi P, Pituch KJ. Sleep problems seldom addressed at two general pediatric clinics. Pediatrics 2001; 107:1375-80

9. Goh DYT, Galster P, Marcus CL. Sleep architecture and respiratory disturbances in children with obstructive sleep apnea. Am J Respir Crit Care Med 2000; 162:682-6.

10. Gozal D, Wang M, Pope DW Jr. Objective sleepiness measures in pediatric obstructive sleep apnea. Pediatrics 200 I; | 08:693-7.

II. Chervin RD, Dillon JE, Bassetti C, Ganoczy DA, Pituch KJ. Symptoms of sleep disorders, inattention, and hyperactivity in children. Sleep 1997;20: I | 85-92.

12. Gozal D. Sleep-disordered breathing and school performance in children. Pediatrics 1998; 102:616-20.

13. Blunden S, Lushington K, Kennedy D, Martin J, Dawson D. Behavior and neurocognitive performance in children aged $5-10$ years who snore compared to controls. J Clin Exp Neuropsychol 2000;22:554-68.
14. Nieminen $P$, Löppönen $T$, Tolonen $U$, Lanning $P$, Knip $M$, Löppönen $H$. Growth and biochemical markers of growth in children with snoring and obstructive sleep apnea. Pediatrics 2002; 109:e55.

15. American Academy of Pediatrics. Clinical practice guideline: diagnosis and management of childhood obstructive sleep apnea syndrome. Pediatrics 2002; 109:704- 12.

16. American Thoracic Society. Standards and indications for cardiopulmonary sleep studies in children. Am J Respir Crit Care Med 1996; I 53:866-78.

17. Souza BB, Hennemann GV, Anselmo-Lima WT. Importance of nasal fiberoptic examination in the presence of a normal $\mathrm{X}$-ray of the cavum. Int J Pediatr Otorhinolaryngol 2000; 55:29-32

Artigo recebido: 13/1 1/04 Aceito para publicação: 05/07/04

\section{Arte Brasileiva}

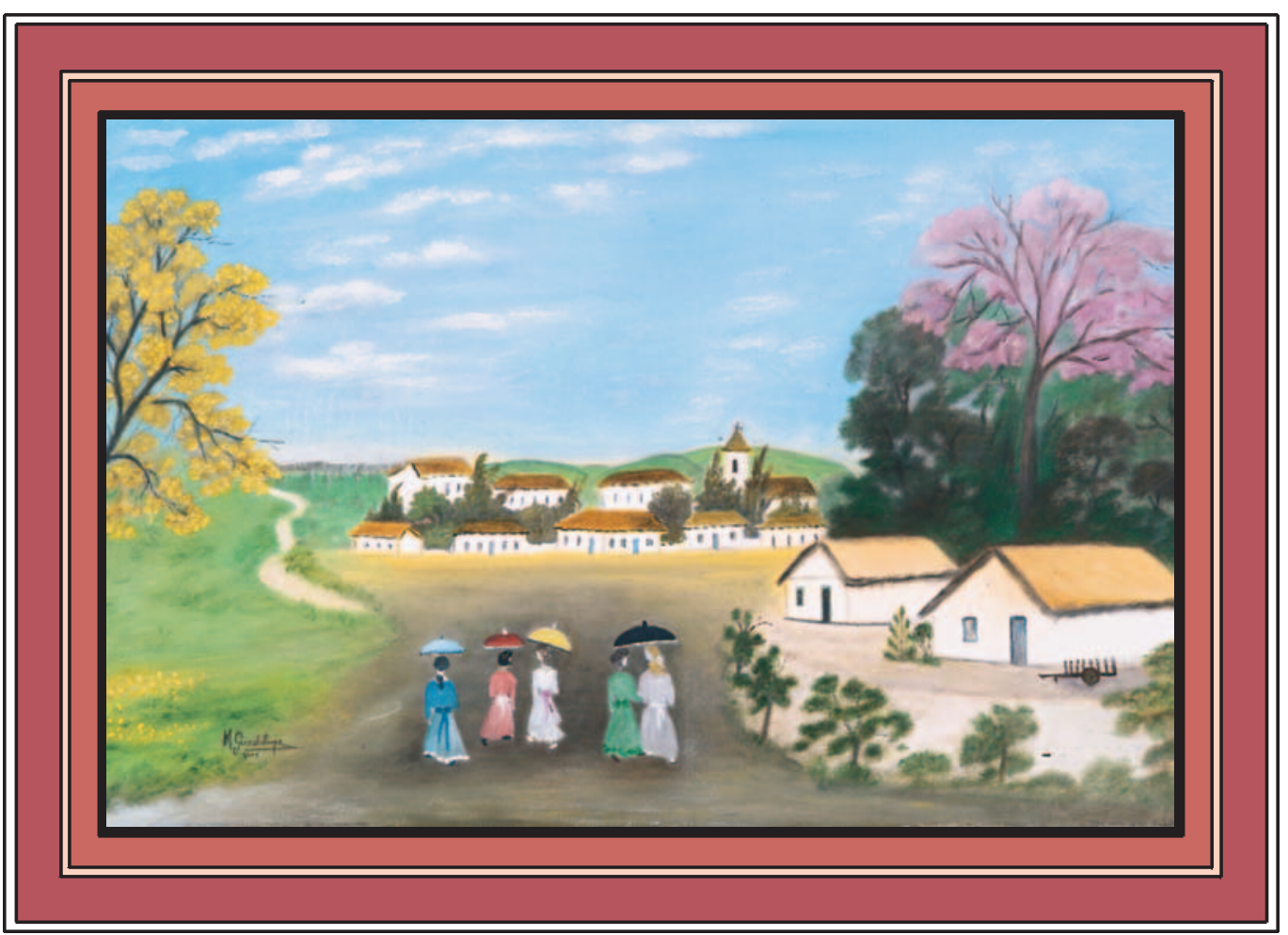

M. Guadalupe - "O passeio das meninas" - Galeria Jacques Ardies - Tel.: (II) 3884-29I6 